\section{La promoción de la investigación en salud pública: búsqueda del equilibrio entre pertinencia y excelencia}

Rebecca de los Ríos ${ }^{1}$
La investigación en salud pública tiene como objetivo fundamental indagar, analizar y explicar la distribución del estado de salud de las poblaciones, los factores que lo determinan, y las respuestas organizadas socialmente para hacer frente a los problemas de salud en términos colectivos. Su propósito es, por consiguiente, generar los conocimientos necesarios para entender las causas y factores que influyen en las condiciones de salud con una perspectiva poblacional, así como evaluar y explicar el efecto que ejercen en dichas condiciones las diferentes políticas, intervenciones y mecanismos de organización de sistemas y de prestación de servicios de salud.

Por su propio carácter, la investigación en el campo de la salud pública se desenvuelve en un terreno de conflictos reales y potenciales que han sido objeto de análisis y reflexión. El conciliar el valor de la excelencia exigida por la comunidad científica y la pertinencia con respecto a las necesidades y resolución de los problemas de salud que exigen los decisores es uno de los conflictos que con mayor frecuencia deben encarar los gerentes y administradores de la actividad científica en el campo de la salud (1). En la búsqueda del equilibrio entre excelencia y pertinencia es necesario asumir varios desafíos, entre ellos el de armonizar los tiempos científicos para la producción de conocimientos y los tiempos políticos para la toma de decisiones; el de inducir la oferta de la comunidad científica en función de las necesidades de salud de las poblaciones, y el de articular mecanismos de diálogo y comunicación entre investigadores y decisores. En los países en desarrollo estos desafíos se tropiezan con las barreras inherentes al nivel de desarrollo de sus infraestructuras científico-tecnológicas y a la competencia de sus investigadores, aspectos que también tienen un impacto directo en la excelencia de la producción científica de dichas naciones. Estos problemas, que se ven hoy en día acrecentados por las restricciones cada vez mayores del financiamiento público destinado a la investigación en materia de salud (2), hacen necesario intensificar el papel activo de abogacía de la comunidad científica para que el conocimiento en el campo de la salud se considere un bien público y la investigación sobre la salud una inversión para el desarrollo.

El propósito de este artículo es describir las formas y mecanismos que se han adoptado en América Latina para buscar el adecuado equilibrio entre la excelencia y pertinencia de la investigación 
en el campo de la salud, y particularmente de la salud pública, haciendo especial hincapié en los mecanismos inductores de la oferta de la comunidad científica a fin de responder preguntas relevantes y pertinentes sobre la solución de los problemas de salud de sus poblaciones. Se exponen, con un breve recuento histórico, los enfoques dominantes en América Latina en las tres últimas décadas y se revisan las modalidades actuales de promoción de la investigación. También se describen en mayor detalle los estudios multicéntricos sobre temas relevantes de salud pública bajo los auspicios del Programa de Subvenciones para la Investigación de la OPS y se destacan los principios que fundamentan un nuevo modelo de colaboración entre los países de la Región, con miras a generar conocimientos orientados a resolver los problemas de salud de las naciones en desarrollo del continente.

\section{Mecanismos para orientar la producción científica en función de las necesidades de salud poblacionales}

Las alternativas propuestas en América Latina para afrontar los desafíos que plantean la pertinencia y relevancia de la producción científica se basaron en la definición de marcos de referencia y de prioridades de investigación en función de planes y programas de desarrollo económico y social. Este enfoque de la planificación de la ciencia, que tuvo su auge en la década de los setenta bajo el liderazgo de los Consejos Nacionales de Ciencia y Tecnología, no logró la enunciada articulación institucional entre la investigación científica y la producción de bienes y servicios para la población (2). En el campo de la salud varios informes evaluativos identificaron y documentaron en los países en desarrollo la creciente brecha entre las necesidades de salud y la inversión en investigación orientada a la solución de problemas (3).

Los indicadores de la producción científica en materia de salud en América Latina durante los años setenta y ochenta muestran un mejoramiento importante de la calidad (excelencia) de dicha producción, pero no así de su pertinencia con respecto a las necesidades de salud. El aumento de la publicación de artículos basados en investigaciones clínicas y básicas siempre superó al aumento del número de artículos publicados sobre investigaciones en salud pública. Si bien en el transcurso de los años ochenta se apreció un ligero incremento de la fracción ocupada por la producción científica en salud pública (4), dicho incremento no representó un verdadero cambio de las tendencias observadas en la producción científica en salud de los países de América Latina.
Este fenómeno ha tenido varias interpretaciones y explicaciones, entre ellas la resistencia de la comunidad científica ante la influencia y participación creciente del Estado y de los planificadores en la elaboración de las políticas científicas (5). Cabe destacar, sin embargo, que los ejercicios de planificación de la actividad científica no llegaron a establecer mecanismos eficaces para la asignación de recursos que obedecieran a criterios de pertinencia con respecto a los planes de desarrollo económico y social. Los Consejos Nacionales de Ciencia y Tecnología de los países de América Latina dedicaron mucho tiempo a tratar de estimular y promover la autonomía y creatividad de los investigadores, dando prioridad a la excelencia y al apoyo de los grupos de investigación. Este período se caracterizó por la recepción de proyectos espontáneos que reflejaban las iniciativas e intereses de los investigadores, y por el predominio de un sistema de evaluación por pares basado en el mérito científico de los proyectos. Ello trajo como consecuencia un fortalecimiento de la oferta y un aumento de la producción científica de excelencia, sin que se lograse la deseada articulación con las necesidades y problemas de salud pública en distintos niveles.

En los años noventa se han dado profundos cambios de alcance mundial y regional caracterizados por la creciente globalización de las economías, fenómeno que impone cambios en la organización de la actividad científica en materia de salud. Aparecen asimismo nuevos actores vinculados con los procesos de desarrollo científico-tecnológico y surgen cambios en los perfiles de salud de las poblaciones y de la organización de los sistemas y servicios de salud. En este nuevo escenario se requieren respuestas novedosas a problemas antiguos y nuevos si se ha de encarar el desafío del equilibrio adecuado entre pertinencia y excelencia.

Una de las estrategias más recientes ha consistido en elaborar y desarrollar las llamadas "agendas concertadas" para la investigación en salud. Las agendas concertadas permiten introducir un marco orientador para racionalizar los recursos en función de los problemas de mayor pertinencia con respecto a las necesidades de salud, pero, a diferencia del enfoque basado en la planificación centralizada, dichas agendas expresan el consenso entre los diversos actores que intervienen en la producción y utilización de los conocimientos en el campo de la salud. Según este enfoque, se concibe la participación del Estado no como ente hegemónico y responsable casi exclusivo de la planificación y financiamiento de la investigación, sino como ente facilitador de consensos y movilizador de los recursos organizativos, políticos y financieros que permitan poner la agenda en ejecución. 
La formulación de las agendas ha variado según el nivel de aplicación - regional, nacional, local- y según el impacto -a corto, mediano y largo plazo- que pretenden tener dichas agendas en la resolución de los problemas de salud y en el propio desarrollo científico-tecnológico de las naciones. A pesar de que se avanza en los mecanismos para la elaboración de las agendas, estas no siempre logran concretarse en proyectos de investigación y desarrollo científico-tecnológico con el financiamiento necesario. Si bien es cierto que las agendas han contribuido en lo metodológico a concertar políticas científicas y de investigación en salud, es preciso diseñar estrategias para su ejecución que se orienten, mediante mecanismos claramente definidos, a generar el impacto deseado en términos de la excelencia y pertinencia de la producción científica.

Conscientes de que el éxito o fracaso de la ejecución de las agendas depende de todo un conjunto de factores, nos limitaremos a analizar los mecanismos y las estrategias de promoción y apoyo a la investigación en salud pública, haciendo especial hincapié en América Latina.

La experiencia de los consejos de ciencia y tecnología durante los años setenta y posteriormente durante la crisis de los ochenta llevó a un replanteamiento de la visión de la ciencia como actividad entre pares y resaltó la necesidad de incorporar a los "no pares" en el proceso de definir políticas y estrategias de desarrollo científico-tecnológico (6). El nuevo reto para lograr un equilibrio adecuado entre la excelencia y la pertinencia de la investigación en salud en América Latina consistió, a partir de entonces, en la búsqueda de mecanismos eficaces para organizar la actividad científica dentro de un contexto de globalización caracterizado por grandes faltas de equidad, tanto en los perfiles de salud como en el acceso y la utilización de los servicios.

Uno de esos mecanismos, como ya hemos visto, es la formulación de las agendas concertadas. No obstante, dichas agendas por sí solas no logran articular procesos para organizar la actividad científica y movilizar a los actores interesados en la producción y en la utilización de los conocimientos. Para que las agendas cobren vida y puedan cristalizarse, es necesario poner en práctica estrategias de promoción y financiamiento de la investigación que permitan movilizar tanto los recursos científicos, como los recursos políticos, organizativos y financieros en los niveles nacional e internacional.

Pese a su diversidad, estas estrategias tienen en común una tendencia a inducir la oferta de la comunidad científica, procurando que los grupos de investigadores respondan a los criterios de pertinencia definidos en las agendas. En los países en desarrollo las estrategias de promoción y financia- miento de la investigación exigen, además, un mejor nivel de excelencia competitiva y por lo tanto obligan a considerar mecanismos que faciliten el fortalecimiento de la investigación y su relación con los centros de excelencia en países desarrollados.

Los mecanismos de inducción que se describirán más adelante también han producido cambios en los sistemas de evaluación, entre ellos el de incorporar en una de las fases de la evaluación de los proyectos a los posibles usuarios o beneficiarios de los resultados de las investigaciones. Asimismo, en los procesos de arbitraje de proyectos se han incluido los criterios de pertinencia con respecto a las prioridades definidas en las agendas, los criterios de relevancia en función de la magnitud y alcance del problema, y los criterios de mérito y excelencia científica.

\section{Los concursos de investigación: garantía de la excelencia por competencia y logro simultáneo de la pertinencia}

Varios han sido los mecanismos inductores de la oferta de los investigadores, entre ellos las convocatorias o concursos de investigación, que son los más frecuentemente utilizados por las agencias nacionales e internacionales de financiamiento. Los concursos son llamados a la comunidad científica para proponer proyectos de investigación basados en los temas de la agenda y de acuerdo con ciertos términos de referencia y requisitos. Estos concursos representan instrumentos que movilizan la capacidad de responder, por medio de la investigación, a la demanda de conocimientos pertinentes o aplicables a la resolución de problemas. Los concursos públicos anunciados por el Consejo Nacional de Ciencia y Tecnología (CONICIT) de Venezuela en el marco de la Agenda "Salud de las Fronteras" (7) han figurado entre los mecanismos concretos que facilitan la búsqueda del equilibrio entre excelencia y pertinencia. Los consejos de ciencia y tecnología de Brasil, México y Costa Rica han seguido procesos similares de inducción (8). En el ámbito internacional, las agencias financieras y de cooperación para el desarrollo también han utilizado este mecanismo para promover y financiar la investigación en salud pública.

En síntesis, los denominados concursos de investigación han sido una de las estrategias de inducción utilizadas con mayor frecuencia en la década de los noventa por organismos nacionales e internacionales de financiamiento a la investigación en salud en América Latina. Aunque no se cuenta con suficientes pruebas que permitan arribar a conclusiones sobre el impacto de estas estrategias en la producción científica de excelencia, no cabe duda 
que los concursos han movilizado y orientado la oferta de la comunidad científica y los recursos destinados a la investigación hacia determinados temas de interés para la salud pública y hacia nuevas áreas que exigen mayores conocimientos. Al mismo tiempo han estimulado la creatividad, la innovación metodológica y la identificación de grupos con la capacidad real y potencial de dedicarse a la investigación en los países de América Latina.

Pese a lo antedicho, esta estrategia ha mostrado sus limitaciones a la hora de formar y consolidar grupos de investigación y de articular redes de colaboración entre ellos y con los centros de excelencia en países desarrollados. Los grupos muchas veces son creados "artificialmente" para satisfacer los requisitos de los concursos y, una vez finalizados los proyectos, dichos grupos se disgregan o dejan de mantener una continuidad en su producción científica. Asimismo y por su propia naturaleza competitiva, los concursos de investigación han tenido poco impacto en el fortalecimiento de la capacidad de investigación en los países de América Latina que exhiben un menor desarrollo de su infraestructura científica y de investigación. Por ende, han tenido también un alcance limitado para lograr este propósito. Finalmente, y por tratarse de una estrategia que resalta el apoyo de ideas o proyectos individuales sin conexión e interdependencia mutua, los mecanismos de comunicación y diseminación de resultados dependerán en gran medida de las iniciativas y capacidades propias de los investigadores.

\section{Concursos para proyectos de investigación en salud pública con el fortalecimiento de capacidades de investigación institucionales}

Los concursos para proyectos de investigación han sido una variante de los concursos anteriormente descritos. La selección se basa en la pertinencia y relevancia de los temas propuestos y el proyecto se constituye en un instrumento que fortalece las capacidades de investigación y de articulación con los niveles de los servicios de salud. Esta estrategia fue impulsada y puesta en práctica con bastante éxito en América Latina y otras partes del mundo por el Programa Especial de Investigación en Enfermedades Tropicales (TDR) de la Organización Mundial de la Salud (OMS). Su apoyo a la producción de conocimientos de excelencia por medio de un asesoramiento técnico directo en la ejecución y publicación de sus resultados se suma a la formación de investigadores, la inversión en infraestructura tecnológica para la investigación, y la incorporación de los resultados de las investigaciones a los programas de control y prevención de enfermedades tropicales. Esta estrategia ha facilitado también la colaboración entre países y ha dado lugar, dondequiera que el TDR ha tenido presencia, a una comunidad científica con gran influencia en el proceso decisorio de los programas para el control de las enfermedades tropicales.

\section{Los proyectos de colaboración entre norte y sur}

Estos proyectos de investigación son también una variante de los concursos de investigación orientados a dar respuesta a preguntas de alta pertinencia a problemas de salud pública de los países en desarrollo, a través del cual se promueve y facilitan la cooperación y el intercambio entre centros de alta excelencia científica - por lo general en países desarrollados- y centros académicos y de investigación de países en desarrollo. Esta estrategia fue impulsada con éxito en los países de América Latina mediante el Programa Conjunto de la Fundación Fogarty de los Institutos Nacionales de Salud, Estados Unidos, y la Organización Panamericana de la Salud (9). El Programa se concentró, sin embargo, en la ejecución de proyectos conjuntos de investigación entre Estados Unidos y América Latina sobre el desarrollo de tecnologías modernas, especialmente en el área de la biotecnología, para la prevención y el diagnóstico de enfermedades de interés para la salud pública en los países de América Latina. Desde más recientemente, la Unión Europea ha contado con un programa de inducción y financiamiento para este tipo de proyectos, dirigido hacia investigaciones cuya orientación primaria es la solución de los problemas de salud de los países en desarrollo (10). Los estudios que concursan también se seleccionan por sus méritos y deben tener un investigador principal de un país europeo y un coinvestigador principal de un país de América Latina o cualquier otro país en desarrollo.

Los concursos de investigación y las variantes señaladas han sido iniciativas que facilitan el equilibrio entre pertinencia y excelencia y que promueven formas de colaboración e intercambio entre centros e investigadores. Sin embargo, los proyectos tienen limitado alcance en cuanto a los temas que deben abordar y, sobre todo, en cuanto a sus posibilidades de generar conocimientos de alta pertinencia para resolver los problemas que son comunes a varios países.

\section{Los proyectos multicéntricos: nuevo modelo de investigación colaborativa en salud pública para la solución de problemas comunes}

En el pasado y en la actualidad se han impulsado proyectos de tipo multicéntrico con el propó- 
sito de producir conocimientos sobre problemas de salud pública que son comunes a varias naciones y que requieren esfuerzos colaborativos, tanto para la investigación como para la intervención. En estos proyectos, en los que participan grupos de investigadores de varios países, el problema y la pregunta central de la investigación se exploran con una metodología única que se aplica a diferentes sujetos o casos en estudio y que permite sacar conclusiones a partir de análisis comparativos en diferentes contextos. No obstante, en los estudios multicéntricos impulsados en América Latina y otros lugares del mundo en desarrollo, los países han participado fundamentalmente como "recolectores de datos", mientras que la idea original, el protocolo, el análisis y la publicación de los resultados han estado en manos de investigadores afiliados con centros de excelencia en países desarrollados. Este modelo, además de reforzar la desventaja competitiva de los países en desarrollo en materia de investigación, dificulta la transferencia de conocimientos $y$, por ende, su utilización y aplicación para la solución de los problemas de salud.

Con el objeto de revertir esta tendencia y redefinir los términos aplicables a la participación de los centros e investigadores de América Latina en este tipo de estudios, el Programa de Subvenciones para la Investigación de la OPS (PSI) ha puesto en práctica un nuevo modelo para la coordinación y ejecución de proyectos multinacionales (11). Estos estudios representan un mecanismo inductor de la oferta de centros de excelencia en la Región y un esfuerzo de colaboración entre países encaminado a producir y diseminar conocimientos para la solución de problemas de salud pública de alcance regional.

Cinco principios orientan los estudios multicéntricos según el nuevo esquema de colaboración que promueve la OPS:

- Selección de problemas de salud pública que afectan a varios países o a la Región como un todo

La selección de los temas y preguntas de investigación tienen como marco las políticas y prioridades de actuación de la Organización y deben responder a las necesidades de conocimientos que permitan resolver los problemas de salud pública de la Región, con particular énfasis en las poblaciones de América Latina y el Caribe. Además de aportar nuevos conocimientos y de abrir camino para otros estudios e investigaciones, los estudios de este tipo deben ofrecer pruebas para fundamentar las políticas e intervenciones. Bajo esta modalidad se selecciona anualmente un problema que exige un esfuerzo de colaboración en investigación comparativa, que puede abordarse mediante metodologías estandarizadas y que puede producir en el corto plazo resultados aplicables a la resolución de problemas comunes a algunos países de la Región o a todos.

\section{- Trabajo participatorio y producción colectiva de los investigadores}

La formulación, ejecución y publicación de los resultados de los estudios multicéntricos bajo los auspicios del PSI son procesos que se construyen y desarrollan con la participación de los investigadores en todas y cada una de sus etapas. La definición del problema y la justificación de los estudios se concretan por medio de proyectos de investigación que compiten según criterios de pertinencia, relevancia, mérito científico y factibilidad técnico-financiera. Acordados los objetivos y el alcance del estudio, se definen la metodología y los instrumentos de recolección de información con la participación de los investigadores. Bajo este principio de construcción y producción colectiva, los estudios multicéntricos responden a los intereses comunes de los países involucrados, y a los responsables de coordinarlos en cada centro o país se les considera los investigadores principales del proyecto en cuestión. El enfoque propuesto por la OPS supera el modelo de conducción y ejecución centralizadas y promueve un esquema basado en la colaboración y participación de los investigadores en todas las fases de los estudios y en igualdad de condiciones.

- Promoción de la colaboración entre países y organización de redes

La formulación y ejecución de estudios multicéntricos exigen la participación de centros de excelencia científica y de grupos de investigadores con experiencia en los temas que se estudian. Esto necesariamente implica la identificación de los centros e investigadores de la Región, la formación de equipos multidisciplinarios y la participación ad-hoc de especialistas, expertos y asesores en las diferentes etapas de los proyectos. De ahí que este tipo de estudio sea una herramienta importante para identificar investigadores que estén trabajando en el tema seleccionado, y para desarrollar redes de colaboración e intercambio entre centros e instituciones de un mismo país y de diferentes países. Finalmente, son un mecanismo para fortalecer la capacidad de investigación en la Región. Se promueven, según este principio, la colaboración y el intercambio entre norte y sur y entre investigadores de los centros y países que participan en los estudios, y se identifican y aprovechan los conocimientos y la pericia que estos pueden aportar al desarrollo de los proyectos en sus distintas etapas. 
- Función de movilizar recursos y promover la responsabilidad compartida

Los estudios multicéntricos se caracterizan por ser proyectos de los países participantes y es responsabilidad de los investigadores movilizar los recursos políticos, organizativos y financieros para garantizar el proceso de colección y análisis de datos y de diseminación y transferencia local de resultados. Los centros e investigadores de los países son responsables de coordinar localmente los proyectos y de poner en práctica procesos de negociación de los recursos complementarios para su ejecución. En esta negociación los investigadores "venden la idea" y se abren los espacios para el diálogo y comunicación entre investigadores y decisores. Asimismo, los investigadores tienen la oportunidad de responder a las demandas de información y de conocimientos de los niveles decisorios y de facilitar la transferencia y utilización de los resultados. Por lo tanto, estos proyectos se conciben como medios para movilizar recursos y articular cada vez más a los investigadores y posibles usuarios de los resultados.

- Papel de facilitación y armonización de la cooperación técnica

Según los principios enunciados anteriormente, se está produciendo un cambio importante en los esquemas de cooperación y en el financiamiento de los proyectos de investigación. De un esquema centralizado para la cooperación y el financiamiento de los proyectos se pasa a un esquema descentralizado en el que se comparte la responsabilidad. La OPS desempeña un papel facilitador en lo referente a la colaboración entre países y es la entidad responsable de armonizar y lograr los consensos para desarrollar el protocolo del estudio y los instrumentos y metodologías estándares que se necesitan para que haya comparabilidad. La OPS también cumple la función de coordinador regional y brinda la cooperación técnica para garantizar la calidad de los proyectos en cada una de sus etapas. Colabora en la formulación del protocolo de investigación, en el diseño y puesta a prueba de los instrumentos de recolección, en los procesos de organización de las bases de datos y en el análisis, publicación y transferencia de los resultados.

De acuerdo con estos principios, el PSI redefine sus políticas y su función se orienta más hacia inducir la oferta, estableciendo nuevos esquemas de colaboración entre investigadores y centros de excelencia de la Región para generar conocimientos y pruebas que sustenten las políticas. Esta labor que realiza con los programas técnicos regionales de la OPS se inició con el Estudio Multicéntrico sobre Normas y Actitudes frente a la Violencia (proyecto ACTIVA), que se realizó en ocho ciudades iberoamericanas y cuyos primeros resultados se publican en este número especial. Es el primer proyecto multinacional que se realiza con esta modalidad de trabajo y fue una importante fuente de aprendizaje y experiencia para la coordinación y gerencia de proyectos de este tipo. Con este esquema de colaboración el PSI continúa promoviendo y apoyando un estudio cada año y hasta la fecha han participado 15 países, 20 centros y cerca de 50 investigadores de América Latina y el Caribe, Estados Unidos y Canadá. Se trata, en síntesis, de una visión y una contribución para el adecuado equilibrio entre la excelencia y la pertinencia de la producción científica en salud en la Región de las Américas, impulsando un nuevo modelo de articulación y colaboración entre países.

\section{SYNOPSIS}

\section{Promoting public health research: balancing relevance and excellence}

Those in charge of directing scientific research face a daunting task: finding a proper balance between excellence in research and that research's relevance to people's health problems. In developing countries, this task is further hampered by deficient scientific and technological infrastructure, the lack of competitiveness of its research community, and the shortage of research funds. This article explains some of the mechanisms that have been put in place in Latin America to achieve a balance between excellence and relevance, especially by promoting research that targets pertinent public health problems. Within this context, the multicenter studies being conducted under the auspices of the Pan American Health Organization's Program for the Support of Research are described. The article also lays out the fundamental features of a new model for international cooperation to generate knowledge to help resolve the public health problems of the countries of the Americas. 


\section{REFERENCIAS}

1. Frenk J. De la pertinencia a la excelencia: dilemas de la investigación en el sector público. Ciencia 1987;38:169-178.

2. Pellegrini A. Bases para la formulación de políticas de ciencia y tecnología en salud en América Latina. Bol Oficina Sanit Panam 1994;116(2): 165-176.

3. Commission on Health Research for Development. Health research: essential link to equity in development. Geneva: COHRED; 1990.

4. Pellegrini Filho A, Goldbaum M, Sivi J. Producción de artículos científicos sobre salud en seis países de América Latina 1973 a 1992. Rev Panam Salud Publica 1997;1(1):23-34

5. Organización Panamericana de la Salud. La investigación en salud en América Latina y el Caribe: tendencias y desafíos.
Presentado en la XXXI Reunión del Comité Asesor de Investigaciones en Salud. Washington, DC: OPS; 1996. (Documento OPS/CAIS96.04).

6. Ávalos I. La ciencia y tecnología en el desarrollo social: el caso de Venezuela. Presentado en la XXXI Reunión del Comité Asesor de Investigaciones en Salud. Washington, DC: OPS; 1996. (Documento OPS/CAIS/96.06).

7. Consejo Nacional de Ciencia y Tecnología. Las políticas de ciencia y tecnología para el desarrollo: el caso de Venezuela. Presentado en la XXXI Reunión del Comité Asesor de Investigaciones en Salud. Caracas: CONICIT; 1998.

8. Organización Panamericana de la Salud. Informe al Director preparado para la XXXI Reunión del Comité Asesor de
Investigaciones en Salud. Caracas: CAIS; 1998.

9. Programa de Investigación y Capacitación en Biotecnología. Organización Panamericana de la Salud y Fundación Fogarty, Institutos Nacionales de Salud de los Estados Unidos. 1995, Washington D.C.

10. European Commission, Science Research Development Directorate-General XII. Health research with developing countries. Bruselas: EC; 1997.

11. Organización Panamericana de la Salud. Evaluación de la Gestión del Programa de Subvenciones para la Investigación de la OPS, 1994-1995: propuesta de estrategia futura. Presentado en la XXXI Reunión del Comité Asesor de Investigaciones en Salud. (Documento OPS/ CAIS/96.01). 